\title{
On Rapid Interstellar Scintillation of Quasars: PKS 1257-326 Revisited
}

\author{
Hayley E. Bignall and Jeffrey A. Hodgson \\ International Centre for Radio Astronomy Research, Curtin Univer sity, WA 6845, Australia \\ email: H.Bignall@curtin.edu.au
}

\begin{abstract}
The line of sight towards the compact, radio loud quasar PKS 1257-326 passes through a patch of scattering plasma in the local Galactic ISM that causes large and rapid, intra-hour variations in the received flux density at centimetre wavelengths. This rapid interstellar scintillation (SS) has been occurring for at least 15 years, implying that the scattering "screen" is at least 100 AU in physical extent. Through observations of the ISS we have measured microarcsecond-scale "core shifts" in PKS 1257-326, corresponding to changing opacity during an intrinsic outburst. Recent analysis of VLA data of a sample of 128 quasars found 6 sources scintillating with a characteristic time-scale of $<2$ hours, suggesting that nearby scattering screens in the ISM may have a covering fraction of a few percent. That is an important consideration for proposed surveys of the transient and variable radio sky.
\end{abstract}

Keywords. techniques: high angular resolution, ISM: structure, quasars: individual

\section{Introduction}

Radio sources of the order of 0.1 milli-arcseconds or smaller in angular size exhibit fluctuations in their measured flux densities at centimetre wavelengths caused by scattering in the Galactic ionised interstellar medium (Rickett, 1990). In the weak scattering régime, which occurs at frequencies above $\sim 4 \mathrm{GHz}$ for most extragalactic lines of sight, the spatial scale of those fluctuations corresponds approximately to the Fresnel scale $r_{\mathrm{F}}$ at the distance of the scattering material, $D ; r_{\mathrm{F}}=\sqrt{D / k}$, where $k=2 \pi / \lambda$ is the wavenumber corresponding to observing wavelength $\lambda$. The corresponding characteristic time-scale of weak interstellar scintillation (ISS) ranges between minutes and days, depending on $\lambda, D$ and $v$, the relative transverse velocity between source, screen and observer. Only the most compact sources scintillate; when the source's angular size, $\theta_{\mathrm{S}}$, significantly exceeds $\theta_{\mathrm{F}}=r_{\mathrm{F}} / D$, scintillation in weak scattering is suppressed owing to averaging over incoherently scintillating regions of the source. Below the transition frequency in the regime of strong scattering, compact quasars will exhibit refractive scintillation on longer time-scales, but are generally too large to exhibit the narrow-band, short-timescale diffractive scintillation displayed by pulsars (Narayan, 1992). The recent MASIV VLA Survey showed that more than half of all compact, flat-spectrum radio quasars exhibit intraday variability due to ISS at $5 \mathrm{GHz}$ with a duty cycle of at least $25 \%$, and rms variations of typically $2-10 \%$ (Lovell et al. 2008). ISS can be used as a probe both of small-scale structure in the Galactic ISM and of source structure on microarcsecond ( $\mu$ as) scales. For further discussion of potential applications, see Koay et al., page 347.

In this paper we discuss the population of quasars which exhibit weak ISS on atypically short time-scales - minutes to hours - on account of nearby Galactic scattering screens. The closer the scattering screen, the smaller $r_{\mathrm{F}}$ and the shorter the characteristic scintillation time-scale for a given velocity, $t_{\mathrm{c}} \sim r_{\mathrm{F}} / v$. In addition, $\theta_{\mathrm{F}}$ is large for small $D$, and 
as quasar angular sizes often exceed $\theta_{\mathrm{F}}$ for $D \gtrsim 10 \mathrm{pc}$ their scintillation will tend to be dominated by nearby scattering plasma. In section 2.1 we present a new analysis of the $\mu$ as-scale evolution of the long-lived rapidly scintillating quasar PKS 1257-326.

\section{2. "Intra-Hour Variable" Quasars}

During the past 15 years three quasars have been found to show large-amplitude ( $\gtrsim 10 \%$ rms) cm-wavelength variability over time-scales $<1$ hour; they are PKS 0405-385discovered with the Australia Telescope Compact Array (ATCA) by Kedziora-Chudczer et al. (1997), J1819+3845, discovered with the Westerbork Synthesis Radio Telescope by Dennett-Thorpe \& de Bruyn (2000), and PKS 1257-326, discovered with the ATCA (Bignall et al. 2003; hereafter B03). That the rapid variability is entirely due to ISS of these sources has been shown by measurements of variability pattern arrival-time delays of typically minutes between widely separated telescopes (Jauncey et al. 2000; DennettThorpe \& de Bruyn, 2002; Bignall et al. 2006, hereafter B06), and by annual cycles in the characteristic variability time-scale (Dennett-Thorpe \& de Bruyn, 2003; B03).

Annual cycles occur as the scintillation velocity (both speed and direction) changes with the Earth's orbit; in this case quasars can be considered fixed on the sky and the velocity of the scattering plasma is of order the same as the Earth's orbital velocity, $\sim 30 \mathrm{~km} \mathrm{~s}^{-1}$. Moreover, the scintillation patterns have been found to be highly anisotropic (Rickett et al. 2002; Dennett-Thorpe \& de Bruyn 2003; B06; Walker et al. 2009). The scintillation time-scale thus increases at times of year when the relative transverse velocity between the Earth and the scattering plasma is small, or more specifically when the velocity component along the minor axis of the scintillation pattern is small. Measurements of scintillation time-scale and two-station pattern arrival-time delays at various times of the year can be used to determine the scattering screen velocity and the length scale, the anisotropy axial ratio and the position angle of the scintillation pattern. The pattern scale also constrains the distance to the scattering screen and the angular size of the source. In practice, in the case of a highly elongated scintillation pattern, the solution becomes degenerate and it may not be possible to constrain all parameters uniquely.

For PKS $0405-385$ and J1819+3845, and also for a number of other intraday variable scintillating sources, the rapid scintillation has been found to be episodic (KedzioraChudczer, 2006; Cimò, 2008). Koay et al. (2011) argue that the episodic ISS of J1819+3845 is possibly related to the nearby scattering "screen" moving out of the line of sight, rather than to intrinsic expansion of the source or to fading of a compact scintillating component. The high anisotropy and intermittency of ISS is suggestive of highly magnetically-stressed structures, of which there is also evidence from pulsar scintillation. The origin of those highly localised scattering screens remains a mystery. Linsky et al. (2008) suggested that scattering may occur in turbulent regions of interaction between nearby partially-ionised warm interstellar clouds, and found cloud velocities consistent with screen velocities determined for background fast scintillators seen through the edges of the clouds. However, there is not yet sufficient evidence to confirm the association.

Recently Koay et al. (2011) searched for rapid scintillation in a sample of 128 flatspectrum quasars and BL Lac objects observed over 11 days with the VLA, detecting 6 rapid scintillators with characteristic time-scales of less than two hours, although none with rms variations larger than $10 \%$. Koay et al. showed that rapid and largeamplitude scintillation requires both relatively nearby screens (within $\sim 250 \mathrm{pc}$ for source sizes of $10 \mu \mathrm{as}$, or within $\sim 12$ pc for larger source sizes of $200 \mu \mathrm{as}$ ) and highly compact sources, i.e., with most of the observed source flux density contained in the scintillating component. 


\subsection{PKS 1257-326 revisited: microarcsecond-scale source evolution}

Although PKS 1257-326 is an otherwise fairly typical flat-spectrum quasar at redshift 1.256, it is unique in that it has shown rapid scintillation in all observations between 1995 and 2011, with a repeating annual cycle in the characteristic time-scale. That implies a scattering structure at least $\sim 100 \mathrm{AU}$ in extent, assuming a minimum velocity for the scattering screen consistent with the annual cycle and time-delay data presented in B06. In ATCA data from 2001 to early 2002, an offset was found between the light curves at 4.8 and $8.6 \mathrm{GHz}$, with the $8.6-\mathrm{GHz}$ light curve consistently leading. The time delay between the two frequencies in those data showed an annual cycle over the first year of the monitoring programme (B03); in subsequent data, however, that offset was not observed. We relate the observed evolution of the frequency offset to long-term intrinsic variability in the inner jet of PKS 1257-326.

Assuming that the scintillation patterns at each frequency are similar and have the same degree of anisotropy, one can calculate the expected time offset by:

$$
\Delta t=\frac{\mathbf{r} \cdot \mathbf{v}+\left(R^{2}-1\right)(\mathbf{r} \times \hat{\mathbf{S}})(\mathbf{v} \times \hat{\mathbf{S}})}{v^{2}+\left(R^{2}-1\right)(\mathbf{v} \times \hat{\mathbf{S}})^{2}},
$$

where $\mathbf{r}$ is the displacement vector between the two frequency components of the scintillation pattern, $\mathbf{v}$ is the scintillation velocity (which is a function of the day of year), $R$ is the axial ratio of anisotropy in the scintillation pattern, and $\hat{\mathbf{S}}$ is a unit vector in the direction of the major axis of the scintillation pattern. This is the same equation used by B06 to calculate the two-station time delay, but with the baseline replaced by the displacement between the scintillation patterns at the two frequencies. In the case of the frequency offset, the displacement vector $\mathbf{r}$ needs to be solved.

Although not uniquely constrained, a reasonable fit to the observed time delay between the 4.8 and $8.6 \mathrm{GHz}$ light curves over the period from February 2001 to February 2002 can be obtained for a displacement vector of $\mathbf{r}(\alpha, \delta)=(-1,2) \times 10^{4} \mathrm{~km}$ (Fig. 1). That assumes a scintillation velocity, pattern axial ratio and position angle corresponding to the preferred annual cycle model given in B06. If we adopt the screen distance $D \sim 10 \mathrm{pc}$ also determined by B06, the displacement corresponds to an offset between the centroids of the 4.8 and $8.6 \mathrm{GHz}$ scintillating components of the source of $\sim 15 \mu \mathrm{as},<0.1 \mathrm{pc}$ at the redshift of PKS 1257-326, with the $4.8 \mathrm{GHz}$ component being displaced to the northwest of the higher-frequency component. Such a displacement is readily explained as an optical-depth effect or "core shift", as has been measured in a number of quasars with VLBI astrometry (Kovalev et al. 2008). The large-scale jet of PKS 1257-326 observed at milli-arcsecond (with VLBI; Ojha et al. 2010) and arcsecond scales (with the VLA; B06) also extends in the same direction. The core shift deduced from the ISS patterns is consistent with synchrotron self-absorption at the base of the jet.

However, data obtained since mid-2002 are consistent with no offset between the two frequency components. By comparison to the long-term changes in mean total flux density and spectral index of PKS 1257-326 (Fig. 2) we see that the period until early 2002 where the frequency offset was observed corresponds to an outburst in total flux density, when the source showed an inverted spectrum between 4.8 and 8.6 GHz. At later times, when there was no offset detected between the two frequency components, the overall spectral index is close to zero. The outburst and core shift are readily explained as due to a region of enhanced emission (probably induced by shock acceleration of particles in the jet) that is initially optically thick at $4.8 \mathrm{GHz}$ owing to synchrotron self-absorption, and later fades and becomes optically thinner. The observed relationship between the offset of the scintillation patterns at different frequencies and the intrinsic source evolution 


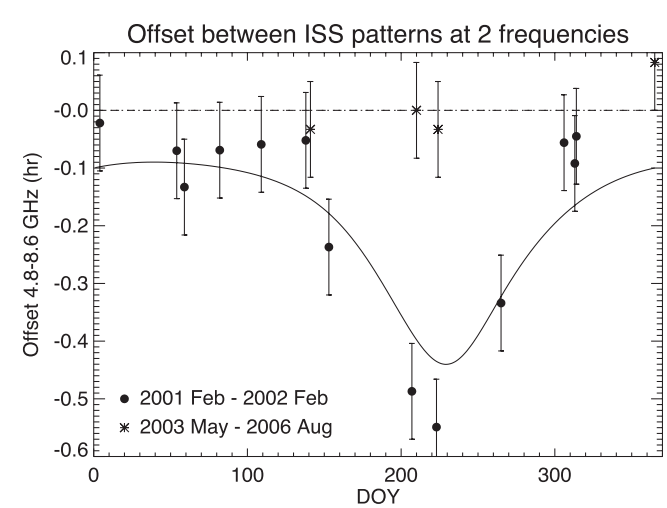

Figure 1. Offset measured by cross correlation of ATCA light-curves at 4.8 and 8.6 GHz. The solid line shows a model annual cycle corresponding to a core shift of $\sim 15 \mu$ as.
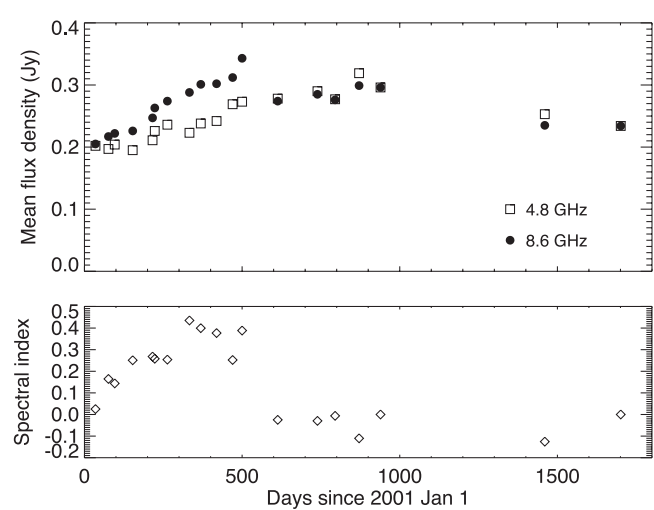

Figure 2. Evolution of average flux density and spectral index over a 5 -year period. The core shift was only observed when the source had an inverted spectrum between 4.8 and 8.6 $\mathrm{GHz}$.

provides strong evidence that the offset is indeed source-intrinsic, and not a refractive effect of the intervening medium.

\section{Summary}

Variability at centimetre wavelengths due to ISS occurs over a range of time-scales. That is an important consideration for proposed surveys for transient and variable radio sources with future wide-field telescopes. Our results for quasar PKS 1257-326 demonstrate the potential of ISS observations at multiple frequencies for studying the physical conditions in otherwise unresolved relativistic jets via the evolution of $\mu$ as-scale structure.

\section{References}

Bignall, H. E., et al. 2006, ApJ, 652, 1050

Bignall, H. E., et al. 2003, ApJ, 585, 653

Cimò, G. 2008, in: The role of VLBI in the Golden Age for Radio Astronomy, Proceedings of Science, PoS (IX EVN Symposium) 046

Dennett-Thorpe, J. \& de Bruyn, A. G. 2000, ApJL, 529, L65

Dennet-Thorpe, J. 2002, Nature, 415, 57

Dennett-Thorpe, J. 2003, A\&\&A, 404, 113

Jauncey, D. L., et al. 2000, in: H. Hirabayashi, P. G. Edwards and D. W. Murphy, Astrophysical Phenomena Revealed by Space VLBI (Sagamihara: ISAS), p. 147

Kedziora-Chudczer, L. 2006, MNRAS, 369, 449

Kedziora-Chudczer, L., et al. 1997, ApJL, 490, L9

Koay, J. Y., et al. 2011, A\&̈A, 534, L1

Kovalev, Y. Y., Lobanov, A. P., Pushkarev, A. B., \& Zensus, J. A. 2008, A\& A, 483, 759

Linsky, J. L., Rickett, B. J., \& Redfield, S. 2008, ApJ, 675, 413

Lovell, J. E. J., et al. 2008, ApJ, 689, 108

Narayan, R. 1992, Phil. Trans. R. Soc. Lond. A., 341, 151

Ojha, R., et al. 2010, A\&A, 519, A45 